\title{
Development of a Fuel Injection RateMeasurement Method for a Vehicle Driven with a Transient Driving Pattern
}

\author{
Choong Hoon Lee \\ Department of Mechanical and Automotive Engineering, \\ Seoul National University of Science and Technology, 232 Kongneungro, Nowon-kuSeoul, 01811, Korea \\ chlee5@seoultech.ac.kr
}

\begin{abstract}
A gasoline fuel injection system constructed with an engine control unit (ECU) was developed to measure the transient fuel injection rate. Various sensor signals from a vehicle driven in the FTP-75 mode with a chassis dynamometer were collected with a data acquisition (DAQ) system. The collected sensor signals were the throttle position sensor (TPS), vehicle speed sensor (VSS), top deadcenter (TDC) position, crank position sensor (CPS) signal, intake air flow rate, and several other sensor signals. Using a FPGA board and a computer, analog voltage signals equivalent to the TPS, TDC, CPS, VSS and air flow sensor signals were reconstructed from the data collected during the chassis dynamometer experiment. All of the reconstructed sensor signals were inputted into the ECU with a synchronized timing scheme. The injectors were connected to the driver pins of the ECU injector. The injectors driven with the ECU output pins were operated under the same condition used in an actual state of a vehicle. The injected fuel quantities were recorded by a mess cylinder and their overall weight was measured with load cells with a time interval of 0.05 seconds. This fuel injection rate measurement system with both an ECU system and load cells showed that the transient fuel injection rate in the FTP-75 mode could be measured successfully. The developed fuel injection system also showed that it can effectively evaluate fuel injection rates in transient operating conditions of a vehicle.
\end{abstract}

Keyword-fuel injection rate, transient mode, engine control unit, FTP-75 mode, loadcell

\section{INTRODUCTION}

In general, there are two fuel injection rate measurement methods for gasoline injectors. One method (the traditional method) [1] records the injected fuel by activating the injector thousands of times with a constant time interval repeatedly, after which it calculates the average injected mass per injection by dividing the recorded total mass by the number of fuel injection instances. Currently, the performance levels of injectors are commonly tested with rigs that are operated using the traditional method. With the traditional method, the fuel injection rate cannot be measured during actual vehicle driving states. The second method (the transient method) measures the accumulated injected fuel mass by driving the injector in a well-defined transient vehicle drive mode, such as the FTP-75 mode [2]. For accurate transient fuel injection measurements, the injector must be driven precisely as it is operated in an actual vehicle driving state. Therefore, the injector must be driven by the ECU, as in a real vehicle. For the ECU to drive the injectors in a realistic vehicle driving state, sensor signals such as the TPS, CPS, TDC, VSS, intake air flow rate, intake air temperature and cooling water temperature signals should be input into the ECU [3].

Previous work related to the transient fuel injection rate measurement method was undertaken by Oh and Lee [4]. Oh and Lee [4] drove the injectors by connecting them to the output pins of an ECU in the FTP-75 mode. The injected fuel quantity was measured with mess cylinders. A camcorder was used to record images of the fuel collected in the mess cylinder according to the elapsed time after the driving of the ECU commenced in the FTP-75 mode. Oh and Lee [4] obtained the fuel injection rate by analyzing the camcorder images. The method of Oh and Lee [4] did not obtain the fuel injection quantity instantly in real time but instead obtained it through image processing after the actual FTP-75 driving experiment. Therefore, their method has problems related to accuracy and timing.

In the present study, to avoid the problems associated with the technique developed by Oh and Lee [4], the injected fuel quantity is measured using load cells, which measure the cumulative injected fuel quantity over time after the start of the fuel injection process as the injectors are driven by the ECU in the FTP-75 mode. By replacing the camcorder with load cells, the injected fuel quantity can be measured instantly at a constant time interval. The fuel injection system developed in this research can be used for evaluating fuel injection rates and fuel economy levels under transient conditions. The fuel injection system as developed here is shown to be effective when used to evaluate fuel injection rates and fuel economy levels under transient operating conditions. 


\section{SENSOR SIGNAL GENERATION TO BE INPUTTO THE ECU}

Generally, the input/output devices of an engine control unit (ECU) consist of several components, including a power unit, microcontrollers and RAM memory, communications link ports (serial port or CAN ports), frequency inputs (e.g., CPS, VSS, TDC sensors), analog inputs (e.g., an air flow sensor, temperature sensor, absolute pressure sensor, TPS signals), PWM outputs (injector or ignition drivers), and frequency outputs (idle speed actuator). The CPS and VSS are used to respectively provide the engine speed and vehicle speed information to the ECU. The CPS, TDC sensor, and VSS generate encoding signals that are processed by the ECU to determine the fuel injection quantity, ignition timing, and transmission shift.
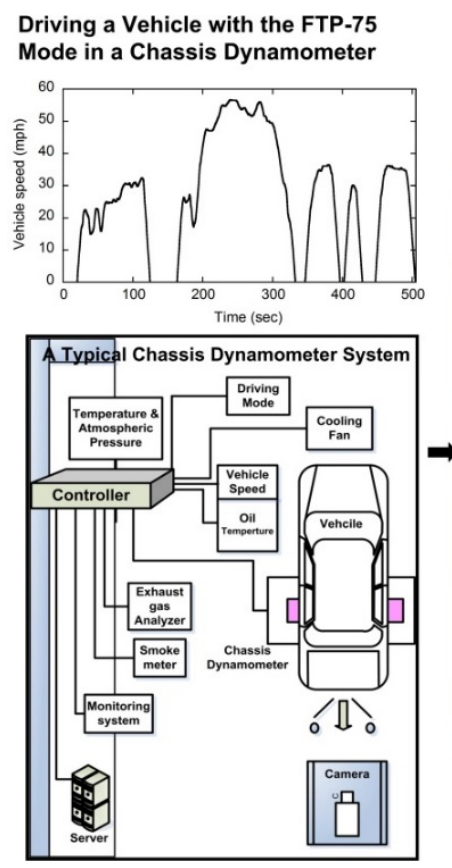

Collected Sensor Data from the Vehicle Driving Reconstruction of the Vehicle Sensor Signals
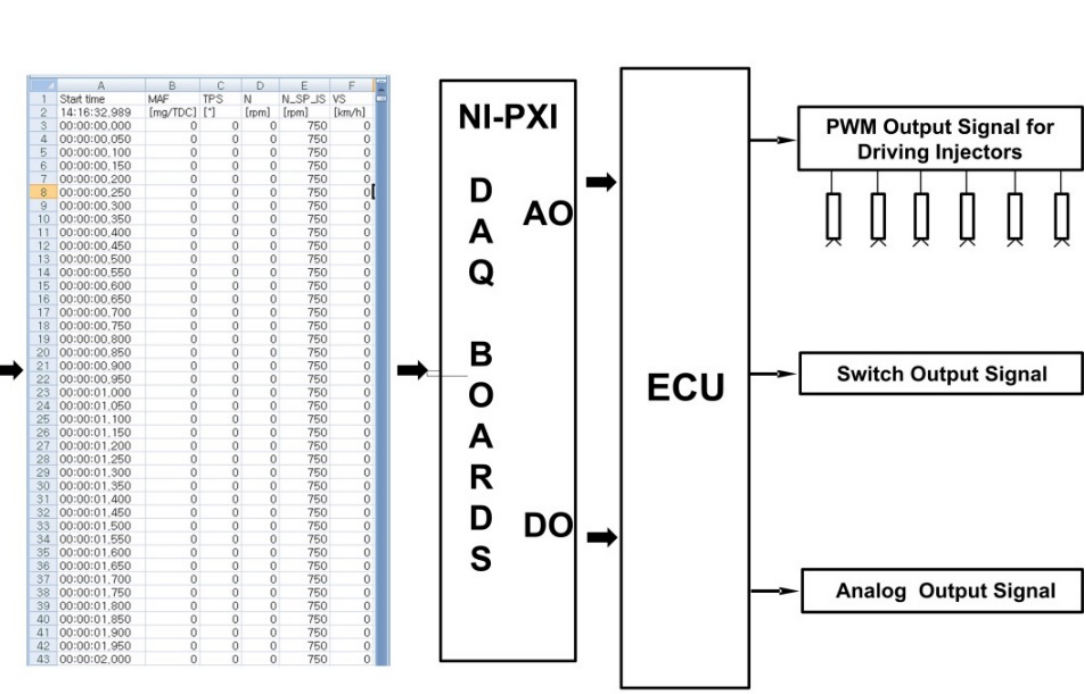

Fig. 1 A schematic diagram of the reconstruction electric sensor signals using the collected sensor data file from the vehicle driven with FTP-75 mode [4]

Fig. 1 shows a schematic diagram of reconstructing electric sensor signals using the collected sensor data file from the vehicle driven with FTP-75 mode (cold transition period) in the chassis dynamometer. The process of reconstructing several sensor signals as input to the ECU consists of two steps. The first step involves the collection of the sensor signals from the vehicle via a chassis dynamometer and saving these signals as data files. That is, the input of the sensor signals to the ECU while the vehicle is driven from the chassis dynamometer is done using a data acquisition (DAQ) system. The second step involves the use of a computer program with DAQ boards to generate electric sensor signals based on the data collected from the files during the first step. LabView ${ }^{\circledR}$ is used for programming [6].

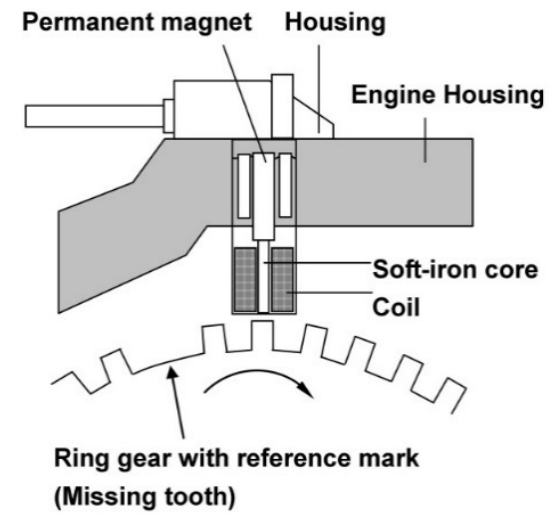

Fig. 2 An inductive type crank position sensor and a ringgear with a reference mark [2] 


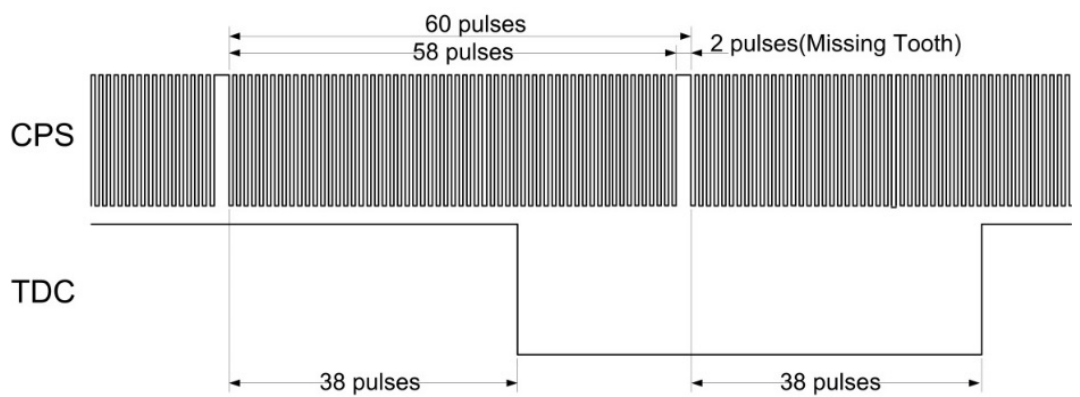

Fig. 3CPS and TDC sensor signals schematics used in this study [4]

The ECU requires the sensor signals along with synchronization of the timing of these signals in order to operate normally. The CPS, TDC sensor, intake air flow rate, VSS, and TPS are supplied to the ECU with synchronized timing. The CPS signal in the vehicle is generated by a Hall element or by a variable reluctance sensor. This signal is used to determine the fuel injection and ignition timing in the engine by combining a TDC sensor and a reference mark (a missing tooth) signal. The typical inductive CPS [2] shown in Fig. 2 consists of a bar magnet with a soft-magnetic pole pin supporting an induction coil with two terminals. When a ferromagnetic ring gear turns past this sensor, it generates voltage in the coil that is directly proportional to the periodic variation in the magnetic flux. The CPS and TDC sensor signals are also reconstructed using the collected data file and DAQ boards. The CPS signal generated in a single revolution of the ring gear of the engine consists of 58 normal pulses, and one reference mark pulse consists of two normal pulses, as shown in Fig. 3. The TDC sensor is also reconstructed to have one pulse for every two revolutions of the engine. The reference mark is used to calculate the stroke position of a specified piston in the engine in conjunction with the signal of the TDC sensor.

The VSS is mounted in the transmission housing and generates four pulses per wheel revolution. The ECU calculates the vehicle speed by counting the frequency of the VSS signal. The frequency corresponding to the vehicle speed can be calculated using Eq. (1). Here, the tire diameter of the vehicle is recorded in the ECU as $687.5 \mathrm{~mm}$. The reconstructed VSS_Frequency DAQ signal is supplied to the ECU.

Frequency_DAQ $Q_{V S S}=(V S S \times 1000000 \times 4) /(\pi \times 687.5 \times 3600)$

The TPS monitors the throttle valve position and travel using a potentiometer. The TPS outputs values of $0.525 \mathrm{~V}$ and $4.475 \mathrm{~V}$ in the idle and WOT (wide-open throttle) conditions, respectively. As the TPS output voltage varies linearly with the open angle of the throttle valve, the relationship between the TPS output voltage and throttle-open angle is expressed as a linear curve fitting, as shown in Eq. (2).

$$
V_{T P S}=0.043 \times T P S_{\text {open_angle }}+0.525
$$

The analog signal of the TPS in the fuel injection equipment was reconstructed using the analog output channel of the DAQ board, which is programmed to the output analog voltage according to the collected TPS voltage while driving the vehicle in the FTP-75 mode.

The air mass flow rate in a vehicle which uses an ECU is measured using a hot-film-type flow sensor [5]. The air flow sensor outputs analog voltage, which increases as the intake air mass flow rate increases. Curve-fitting between the air mass flow rate and the output voltage of the air flow sensor was done in this case using Eq. (3).

$$
V_{\text {AFS }}=\log \left(\dot{m}_{\text {air flow }}\right)-2.203
$$

The analog voltage signal used to simulate the air mass flow rate was reconstructed using the analog output channel of the DAQ board. The cooling water and intake air temperatures are assumed to be constant at $20.3^{\circ} \mathrm{C}$ to simulate the cold transition period of the FTP-75 driving mode. The cooling water and intake air temperatures were reconstructed here with a potentiometer, which generates constant DC voltages corresponding to cooling water and intake air temperatures of $20.3^{\circ} \mathrm{C}$. An ECU scanner was used whether the reconstructed signals were correctly input to the ECU. The scanned results of the TPS, vehicle speed, air mass flow rate, and engine speed show that their signals are dependent on one another. In particular, the dependency between the AFS and engine speed is very high.

\section{III.MEASUREMENT SYSTEM FOR MEASURING THE INJECTED FUEL QUANTITY USING LOAD CELLS}

The method developed by Oh and Lee [4] for measuring injected fuel quantities reads the mess cylinder scale using captured images. This method cannot read and display the injected fuel quantity in real time because the scale of the mess cylinder is obtained by image processing after the experiment. In order to read the injected fuel quantity of six mess cylinders, two camcorders were necessary to capture the mess cylinders' scale. This resulted in inaccurate and time-consuming measurements. 
Fig. 4 shows a schematic diagram of the ECU fuel injection system used to measure the injected fuel quantity using load cells. The experimental system consists of injectors, load cells, the ECU, a fuel pump, two fuel rails, a DAQ system, and a strain amplifier. With regard to the DAQ system, the NI-PXI ${ }^{\circledR} 1042$ system embedded with a NI PXI ${ }^{\circledR}-6251$ and a 6220 board was used for the ECU fuel injection system. Curiotech ${ }^{\circledR}$ load cells with a maximum measurement range of $1 \mathrm{kgf}$ were used. The strain amplifier used in the experiment was the Vishay Precision ${ }^{\circledR}$ signal conditioning amplifier, model 2310. The load cells were calibrated with a set of known reference weights.

FTP-75 for the city driving cycle involves a series of tests defined by the US Environmental Protection Agency (EPA) to measure the tailpipe emissions and fuel economy of passenger cars. Fig. 5 shows the FTP-75 mode for a tested vehicle driven in the chassis dynamometer. The FTP-75 driving mode consists of three phases: a cold start phase (505 seconds), a transient phase (867 seconds) and a hot start phase (505 seconds). The first two phases are run together as one large phase and are followed by stopping the engine for 10 minutes, restarting the engine to test a warm restart, and re-running the first phase again for a complete three-phase FTP75 mode. The total vehicle driving duration, the distance travelled, and the average speed are 1874 seconds, $17.77 \mathrm{~km}$ and $34.1 \mathrm{~km} / \mathrm{h}$, respectively. In this study, the ECU fuel injection system was operated only during the cold start phase.

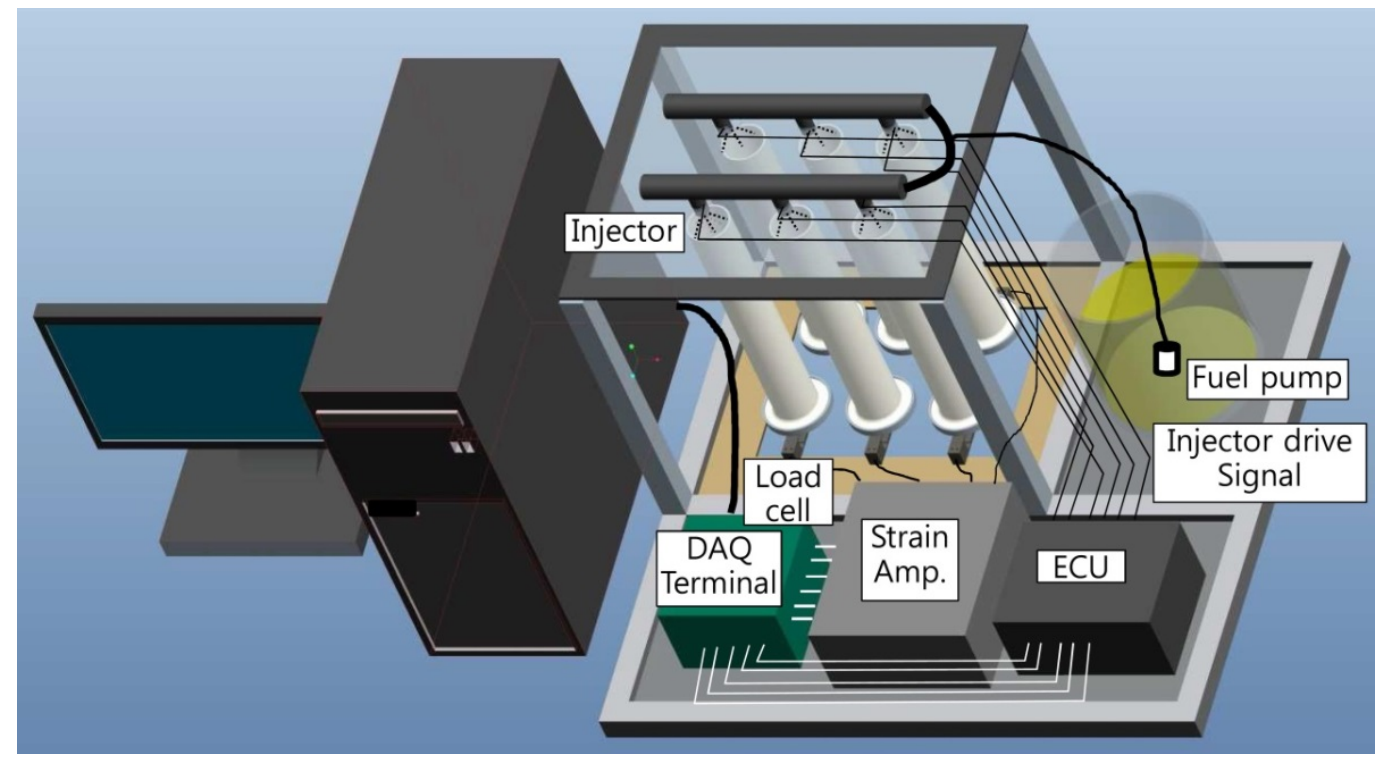

Fig. 4 A schematic diagram for measuring the injected fuel quantity using load cells

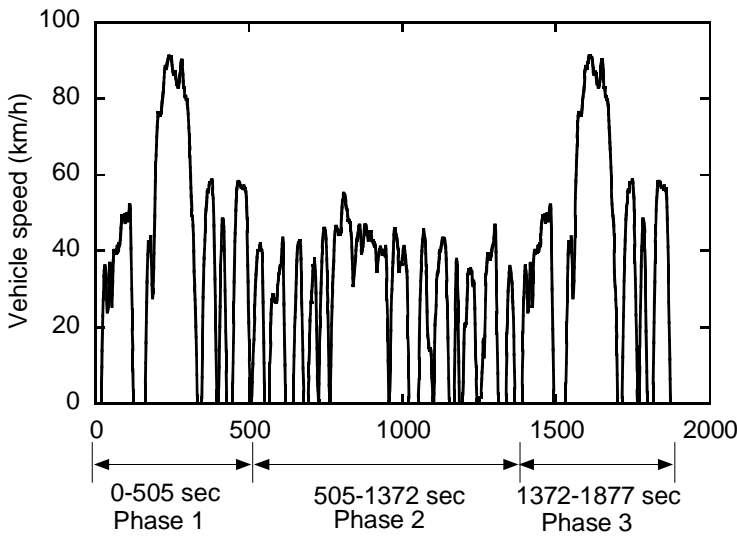

(a)

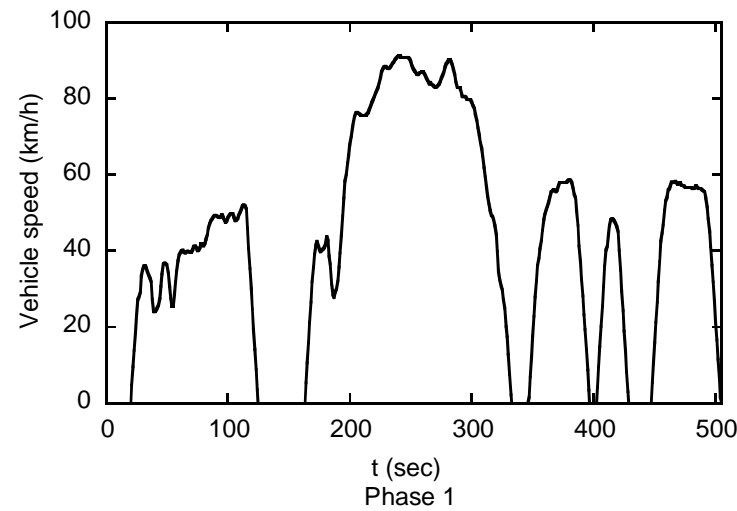

(b)

Fig. 5 FTP-75 driving mode (a) a total of 1877 seconds of the driving phase (b) magnification of the cold start phase 


\section{IV.RESULTS AND DISCUSSIONS}

The ECU fuel injection system using the load cells was developed to identify whether the system could substitute for the actual driving conditions of a vehicle. The fuel supply system, six injectors, and two fuel rails were attached to the developed ECU fuel injection system. After the injectors were connected to the driving port of the injector in the ECU, the ECU fuel injection system was operated during the cold start phase in the FTP-75 mode by supplying the reconstructed CPS, TDC, VSS, AFS (air flow sensor), intake air and cooling water temperature signals, as explained in section II, to the ECU input pins. The fuel quantity injected while driving the vehicle in the FTP-75 mode was recorded with mess cylinders. The weight of the accumulated fuel injected into the mess cylinders were recorded using load cells according to the elapsed time after the driving of the ECU commenced in the FTP-75 mode.

Fig. 6 shows the measured throttle position while driving the tested vehicle in the chassis dynamometer with the cold start phase of the FTP-75 mode. The opening angle of the throttle valve during the cold start phase varied from $0 \%$ to $35 \%$. The variation of the throttle opening angle is completely synchronized with the timing of the air mass flow rate, as shown in Fig. 7. This result is predictable because the air mass flow rate is mostly affected by the opening angle of the throttle valve. As the throttle valve opening angle increases due to acceleration, the air mass flow rate increased, which causes an increase in the engine torque, thus increasing the vehicle speed.

Fig. 8 shows the measured engine speed during the cold star phase of the FTP. The variation in the engine speed with the opening angle of the throttle valve is not highly correlated compared to that of the air mass flow rate and the throttle angle because the engine speed is also affected by the external load of the driving vehicle. The correlation between the opening angle of the throttle valve and the engine speed is stronger when the vehicle accelerates compared to the other driving ranges.

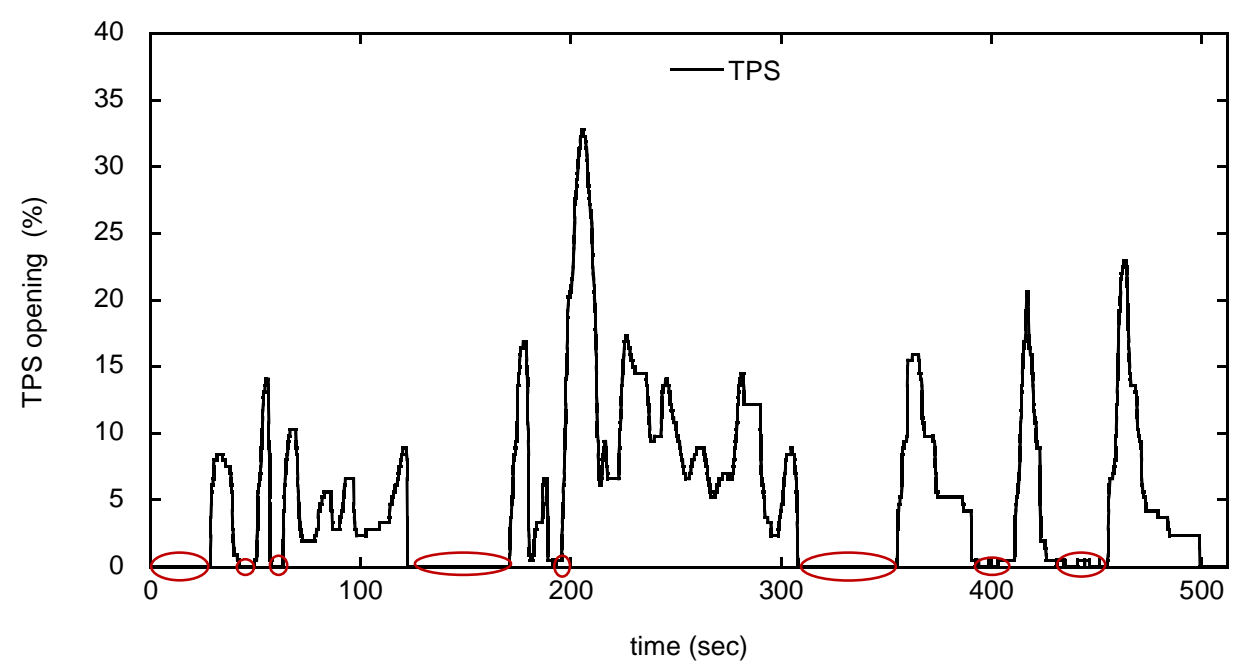

Fig. 6 Measured throttle position sensor signal during the cold start phase of the FTP-75 mode

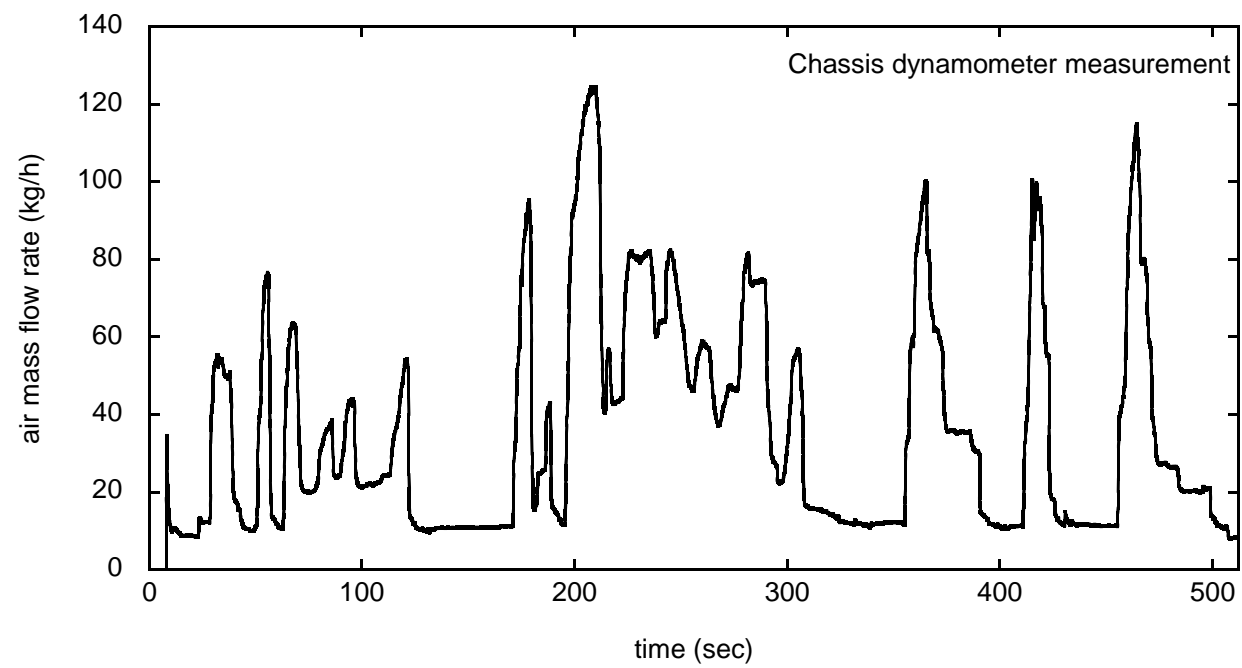

Fig. 7 Measured air mass flow rate during the cold start phase of the FTP-75 mode 


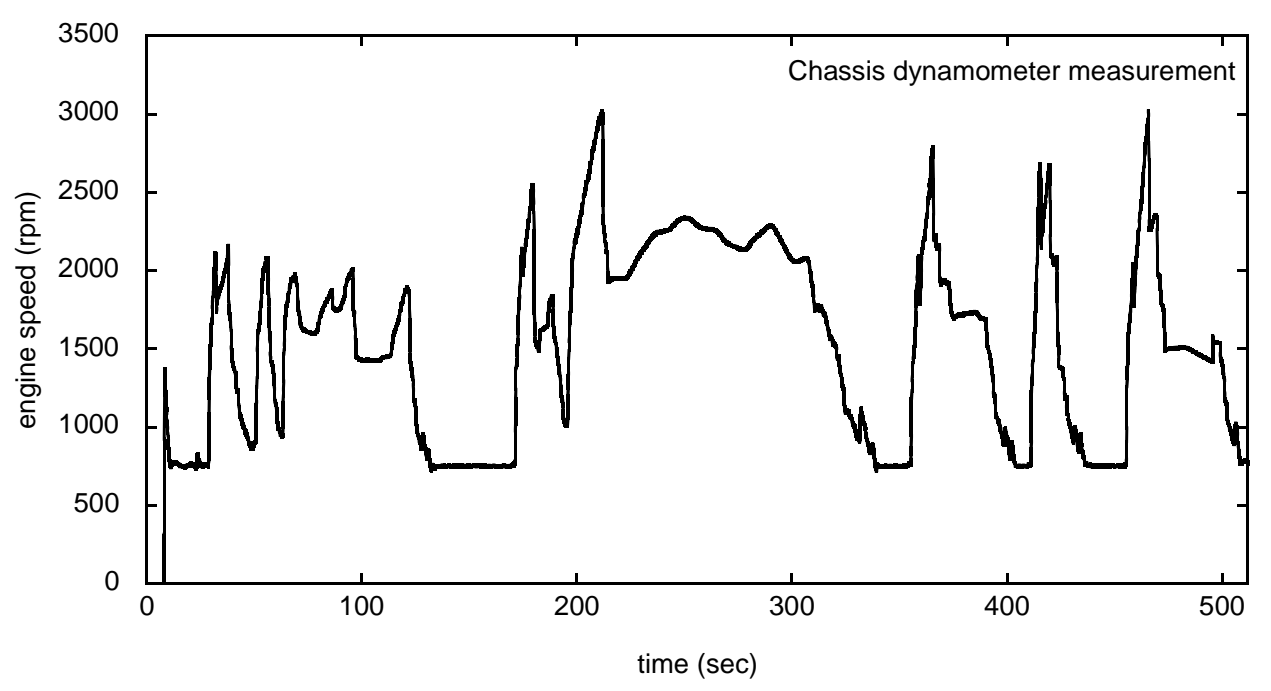

Fig. 8 Measured engine speed during the cold start phase of the FTP-75 mode

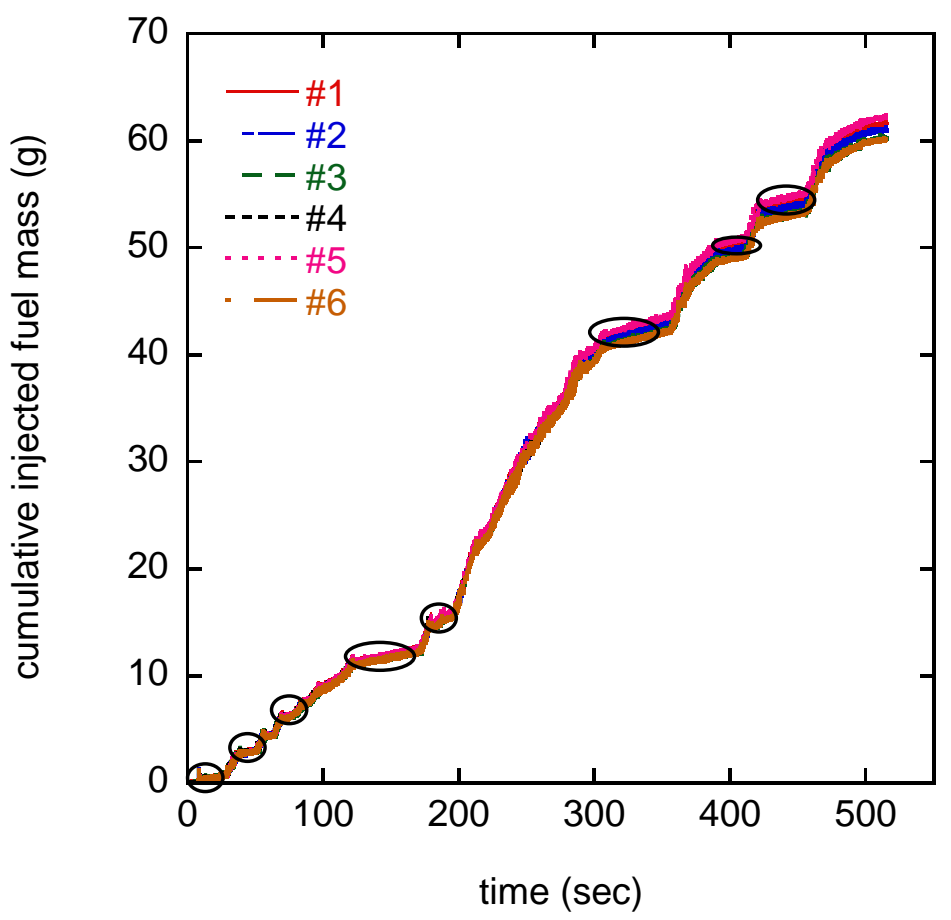

Fig. 9 Measuredcumulative injected fuel quantity during the cold start phase of the FTP-75 mode using load cells

Oh and Lee [4] showed that an ECU fuel injection system and a camcorder can be used to predict the fuel injection quantity of an actual vehicle within 5\% error. Oh and Lee's [4] results from the ECU fuel injection system when used to drive the injectors demonstrated the feasibility of the system.

In this study, load cells replace the camcorder of Oh and Lee [4]. Fig. 9 shows the measured cumulative injected fuel quantities of six gasoline injectors according to time elapsed after the start of the cold start phase. The numbers displayed in Fig. 9 are the injector numbers. The variations of the accumulated quantities of the six injectors when compared to each other are very small, which is vital for good injectors. The variations when using the load cells are reduced compared to those when using Oh and Lee's [4] method, indicating that the measurement accuracy of the injected fuel quantities using these types of load cells can be enhanced. There are several driving ranges with only a slight increase in the cumulative injected quantity curve. The slightly increased time ranges are specific driving time ranges in which the throttle valve opening angle is held at $0 \%$ (engine idle condition). Figure 6 shows that there are eight time range positions (marked with the red circles) where the throttle opening angle is $0 \%$. The eight positions where the throttle opening angle is $0 \%$ correspond to those where the cumulative injected quantity increase slightly, as shown in Fig. 9. The measurement results using the load cells can precisely record the eight specific driving time ranges (as denoted by the black circles) where the curve of the cumulative injected fuel quantity increases slightly. In contrast, the measurement results 
when using the method developed by Oh and Lee [4] cannot pinpoint this range of a slight increase in the cumulative injected fuel quantity curve.

\section{CONCLUSION}

A developed ECU fuel injection system using load cells was assessed to identify whether it could serve as a substitute for the actual driving conditions of a vehicle. The cumulative injected fuel quantity was successfully measured by supplying a reconstructed sensor signal to the ECU based on a data file collected using a dynamometer when a vehicle was driven in the FTP-75 mode. Variations of the measured quantity between each injector using the load cells are reduced compared to the method of Oh and Lee [4]. The measurement results using the load cells can pinpoint exactly eight specific driving time ranges where the curve of the cumulative injected fuel quantity increases slightly due to the idle position of the opening angle of the throttle valve $(0 \%)$.

\section{ACKNOWLEDGMENT}

This study was supported by the Research Program funded by the Seoul National University of Science and Technology

\section{REFERENCES}

[1] Robert Bosch GmbH,Diesel Engine Management, 4th ed.,pp. 68-83,Cambridge, MA, USA: Bentley Publishers,2005.

[2] Robert Bosch GmbH, Automotive Handbook, 7th ed, pp. 582-590, West Sussex, UK: John Wiley \& Sons Ltd, 2007.

[3] J. B. Heywood, Internal Combustion Engine Fundamentals, pp. 296-304, New York, USA: McGraw-Hill, 1988.

[4] D. S. Oh and C. H. Lee, "A study on the fuel injection system simulating a vehicle drivenwith FTP-75 Mode for cold transition period,”Journalof ILASS-KOREA,vol. 16, No. 2, pp., June, 2011.

[5] Robert Bosch GmbH,Gasoline Engine Management, 3rded,West Sussex, UK: John Wiley \& Sons Ltd, 2006.

[6] http://www.ni.com 